УДК 621.315 .592

\title{
Детектор терагерцового излучения на основе термоэлектрического материала $\mathrm{Bi}_{88} \mathrm{Sb}_{12}{ }^{*}$
}

\author{
( Е.С. Макарова ${ }^{1}$, А.В. Асач ${ }^{1}$, И.Л. Тхоржевский ${ }^{1}$, А.Д. Сединин ${ }^{1}$, Д.В. Зыков ${ }^{1}$, А.Д. Зайцев ${ }^{1}$, \\ П.С. Демченко ${ }^{1}$, М.Г. Новоселов ${ }^{1}$, В.А. Комаров ${ }^{2}$, А.С. Тукмакова ${ }^{1}$, \\ А.В. Новотельнова ${ }^{1}$, Н.С. Каблукова ${ }^{1,3}$, М.К. Ходзицкий ${ }^{1}$ \\ ${ }^{1}$ Университет ИТМО, \\ 197101 Санкт-Петербург, Россия \\ ${ }^{2}$ Российский государственный педагогический университет им. А.И. Герцена, \\ 191186 Санкт-Петербург, Россия \\ ${ }^{3}$ Санкт-Петербургский государственный университет промышленных технологий и дизайна, \\ 191186 Санкт-Петербург, Россия \\ E-mail: makarova_helena_2011@mail.ru \\ Поступила в Редакцию 24 сентября 2021 г. \\ В окончательной редакции 20 ноября 2021 г. \\ Принята к публикации 20 ноября 2021 г.
}

Излучение терагерцового диапазона частот перспективно для визуализации, детектирования и передачи данных. Общим для всех этих применений является проблема поиска высокочувствительного, быстродействующего, компактного терагерцового детектора, работающего при комнатной температуре. Поэтому в настоящий момент стоит проблема поиска новых материалов для использования в системах детектирования терагерцового излучения. Значительным потенциалом для решения данной проблемы обладают термоэлектрические материалы на основе твердых растворов висмута и сурьмы. В данной работе впервые исследован отклик в пленках толщиной 70 и 150 нм из термоэлектрического материла $\mathrm{Bi}_{88} \mathrm{Sb}_{12}$ при воздействии терагерцового излучения на частоте 0.14 ТГц.

Ключевые слова: терагерцовое излучение, фотоотклик, висмут, сурьма, термоэлектрические материалы, тонкие пленки.

DOI: $10.21883 /$ FTP.2022.04.52192.7a

\section{1. Введение}

Излучение терагерцового (ТГц) диапазона частот перспективно для решения различных практически важных задач и может быть использовано в системах спектроскопии [1], визуализации [2], детектирования и передачи данных [3]. Для ТГц технологий актуальна проблема, связанная с отсутствием доступных и эффективных терагерцовых устройств [4]. Так, несмотря на то, что за последние два десятилетия значительное количество исследований было посвящено разработке высокочувствительного, быстродействующего, компактного детектора ТГц излучения, работающего при комнатной температуре, в этом направлении остается много нерешенных задач [5]. Одна из основных задач заключается в поиске новых материалов с высокой чувствительностью кТГц излучению при комнатной температуре, для их использования в качестве функциональной среды в системах детектирования. Большинство предлагаемых к настоящему моменту терагерцовых датчиков либо обладают высокой скоростью отклика, но имеют низкую чувствительность, либо об-

* Доклад на XXVII Межгосударственной конференции „Термоэлектрики и их применения“" (ISCTA 2021), Санкт-Петербург, $13-16$ сентября 2021 г. ладают высокой чувствительностью при низком быстродействии [6].

Значительным потенциалом для решения данной проблемы обладают термоэлектрические материалы на основе твердых растворов висмута и сурьмы [7]. Благодаря малой ширине запрещенной зоны они относятся к классу полуметаллов с хорошими термоэлектрическими характеристиками. Поглощение ТГц излучения полуметаллами приводит к возникновению дополнительных пар носителей заряда за счет поглощения энергии, достаточной для перевода электрона в зону проводимости, что аналогично возникновению горячих электронов (дырок) при термоэлектрическом эффекте. Сигнал, возникающий за счет фототермоэлектрического эффекта, возможно усилить. Для этого необходимо использовать материал с максимальной термоэлектрической добротностью для заданной температуры окружающей среды [8].

В данной работе изучается отклик в термоэлектрических пленках $\mathrm{Bi}_{88} \mathrm{Sb}_{12}$ с толщиной 70 и 150 нм при поглощении излучения ТГц диапазона частот разной поляризации в зависимости от расстояния между генератором и детектором. Также анализируется работа детектора при приложении внешнего электрического поля. 

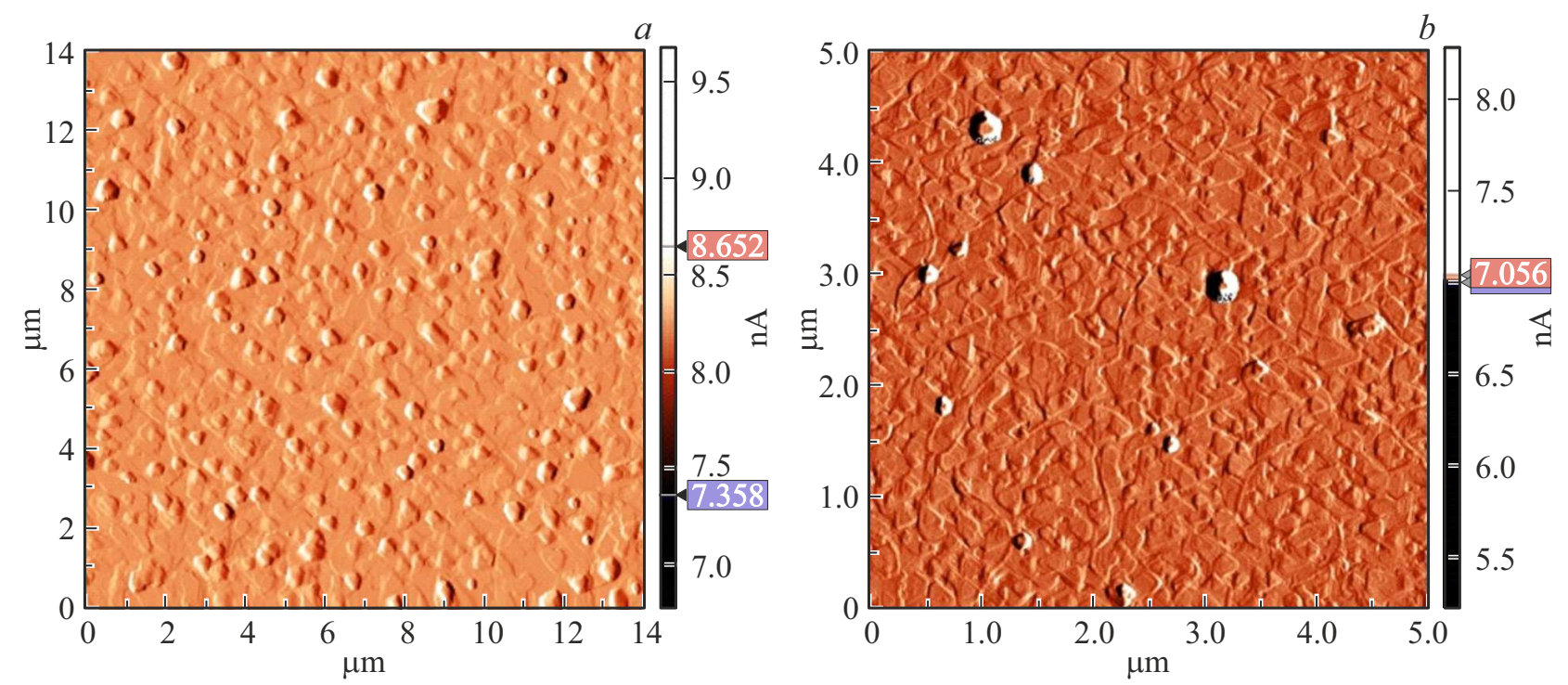

Рис. 1. АСМ-изображение поверхности пленки $\mathrm{Bi}_{88} \mathrm{Sb}_{12}$ на слюде; толщина пленки: $a-150$ нм, $b-70$ нм.

\section{2. Материалы и методы}

\section{1. Подготовка образцов}

Для исследования были получены образцы пленок твердого раствора висмут-сурьма на слюде толщиной 21 мкм. Изготовление пленок производилось методом термического испарения в вакууме. Для получения пленок однородного состава по всему объему использовалось дискретное испарение. Пленки формировались в вакууме при давлении $2 \cdot 10^{-3}$ Па. Все пленки были получены в одинаковых технологических режимах: во время осаждения вещества температура подложки поддерживалась равной $393 \mathrm{~K}$, затем они отжигались непосредственно в испарительной камере под вакуумом при температуре $493 \mathrm{~K}$ в течение 30 мин. В результате были получены пленки твердого раствора висмут-сурьма размерами $0.8 \times 1$ мм, толщиной 150 и $70 \mathrm{HM}$, и концентрацией сурьмы $12 \%$. Толщина пленок измерялась методом оптической интерферометрии с помощью интерферометра Линника МИИ-4 (погрешность измерения не превышает 5 нм). Анализ структуры пленок проводился с помощью метода атомно-силовой микроскопии (АСM). Исследование проводилось на сканирующем зондовом микроскопе Solver P47-PRO в полуконтактном режиме. На рис. 1 представлен рельеф поверхности пленок $\mathrm{Bi}_{88} \mathrm{Sb}_{12}$ толщиной 150 и 70 нм. Пленки имеют два типа неоднородностей: выпуклые трехмерные образования в виде бугорка и треугольные фигуры роста. Треугольная текстура отражает ромбоэдрическую кристаллическую структуру Вi [9]. Такая форма фигур роста указывает на то, что кристаллографическая ориентация этой пленки соответствует направлению оси $C_{3}$ параллельно нормали пленки $[10,11]$, а оси $C_{1}$ и $C_{2}$ лежат в плоскости подложки и они параллельны и противоположно направлены в соседних кристаллитах [12]. По взаиморасположению фигур роста на поверхности пленки определяется размер кристаллитов пленок $[11,13]$ : для пленки толщиной 150 нм размер кристаллитов может достигать 3 мкм (см. рис. 1,a), для пленки толщиной 70 нм размер кристаллитов не превышает 1.5-2 мкм (см. рис. $1, b)$.

\section{2. Ожидаемые эффекты и явления}

При поглощении веществом электромагнитного излучения будет проявляться внутренний фотоэффект, если энергия кванта излучения достаточна для переброса электронов из валентной зоны в зону проводимости. Это условие выполнимо при взаимодействии твердого раствора $\mathrm{Bi}_{88} \mathrm{Sb}_{12}$ с ТГц излучением в широком диапазоне частот от 0.1 до 10 ТГц. В работе [14] было показано, что плазменная частота носителей заряда, определяющая верхнюю частотную границу возможности детектирования излучения, в объемном висмуте лежит в диапазоне от 93 до 99ТГц (в зависимости от поляризации излучения) при температуре $300 \mathrm{~K}$. В случае охлаждения до температуры $80 \mathrm{~K}$ плазменная частота снижается до значений 59-62 ТГц, что хорошо согласуется с результатами работы [15], в которой исследовались особенности спектров отражения легированных кристаллов висмут-сурьма в длинноволновой инфракрасной области спектра. Однако, как было показано в работе [16], при снижении толщины пленки висмута до значений порядка от 40 до 150 нм при температуре $300 \mathrm{~K}$ также наблюдается уменьшение плазменной частоты (по сравнению с объемными образцами) до 55 ТГц при толщине $40 \mathrm{Hм}$ и до 67 ТГц при толщине 150 нм. Следовательно, под воздействием монохроматического 

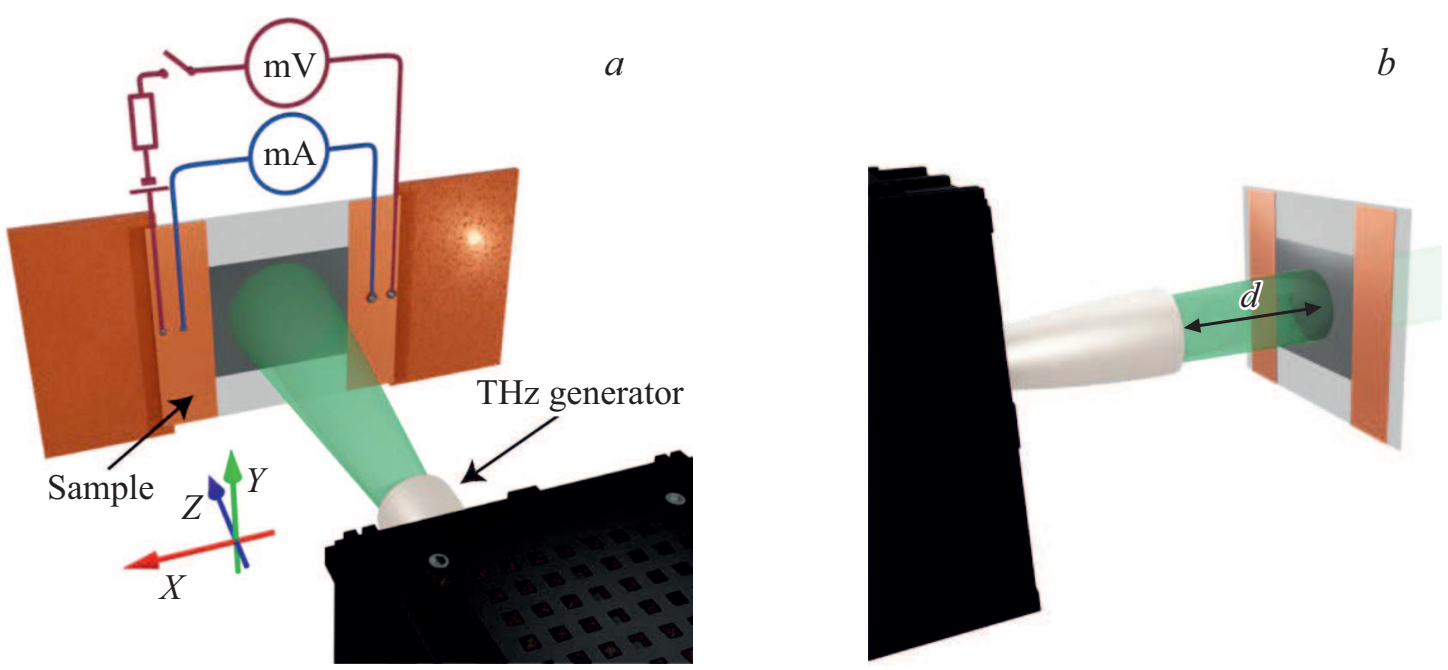

Рис. 2. Схема установки: $a$ - ориентация поляризации и четырехзондовый метод, $b-$ изменение расстояния между источником и пленкой.

излучения 0.14 ТГц в $\mathrm{Bi}_{88} \mathrm{Sb}_{12}$ при $300 \mathrm{~K}$ будет наблюдаться поглощение излучения, и для описания процессов можно использовать теорию внутреннего фотоэффекта для полупроводников. Проводимость пленки может быть описана формулой

$$
\begin{aligned}
\sigma & =\sigma_{0}+\Delta \sigma \\
& =q \mu_{e} n_{0 e}+q \mu_{p} n_{0 p}+\left(q \mu_{e} n_{d e}+q \mu_{p} n_{d p}\right)
\end{aligned}
$$

где $\sigma_{0}$ и $\Delta \sigma$ - темновая проводимость и фотопроводимость, $n_{0 e}$ и $n_{0 p}$ - концентрации электронов и дырок соответственно, $n_{d e}$ и $n_{d p}$ - концентрации генерируемых излучением электронно-дырочных пар, $\mu_{e}$ и $\mu_{p}$ подвижность зарядов, $q$ - модуль заряда электрона.

\section{3. Методика проведения эксперимента}

В данной работе были проведены эксперименты по исследованию отклика в пленках твердого раствора $\mathrm{Bi}_{88} \mathrm{Sb}_{12}$ при поглощении терагерцового излучения. Исследовалось влияние величины приложенного напряжения, угла поляризации падающего излучения, и расстояния от источника излучения до пленки на величину внутреннего фотоэффекта. Суть эксперимента заключалась в последовательном фотовозбуждении неравновесных носителей заряда. В качестве источника терагерцового излучения использовался коммерческий источник непрерывного излучения с частотой 0.14 ТГц и мощностью 30 мВт марки Terasense. Индуцированная терагерцовым излучением фотоэдс регистрировалась с помощью четырехзондового метода (рис. 2). От источника постоянного напряжения $1.5 \mathrm{~B}$ через образец и магазин сопротивлений до 5 кОм подавался ток, не приводящий к нагреву образца. Измерения проводились при двух ориентациях поляризации вектора напряженности электрического поля ТГц излучения: параллельно и перпендикулярно направлению протекания тока. $\mathrm{B}$ первой серии экспериментов тонкие пленки $\mathrm{Bi}_{88} \mathrm{Sb}_{12}$ исследовались под воздействием терагерцового излучения с протекающим постоянным током вдоль пленок. Фотоотклик регистрировался милливольтметром Keithley DMM7510.

Во второй серии экспериментов, вследствие изменения расстояния $(d)$ между рупорной антенной источника ТГц излучения и поверхностью пленки, была зарегистрирована стоячая ТГц волна. Так как стоячая волна возникает из-за множественных переотражений между рупором и образцом, предполагается, что отклик, измеряемый милливольтметром пропорционально мощности излучения, будет меняться от расстояния между образцом и источником. Таким образом, при увеличении $d$ от 0 до 1500 мкм возникают несколько минимумов и максимумов значений мощности сигнала с периодом $\lambda / 2$ (где длина волны $\lambda \approx 2141$ мкм).

\section{3. Результаты}

На рис. 3 и 4 представлена характерная зависимость падения напряжения на образце при отсутствии и наличии излучения. Для пленок поддерживалось постоянное значение силы тока: для пленок толщиной 150 и 70 нм ток составлял $0.107 \mathrm{MA} \mathrm{и} 0.010 \mathrm{MA}$.

На рис. 3, $a$ и 4, $a$ для пленки толщиной 70 нм приведены две зависимости напряжения от времени, которые показывают влияние поляризации излучения. На рис. $3, b$ и $4, b$ предоставлена зависимость падения напряжения в пленках при перпендикулярном направлении поляризации излучения току, проходящему через пленку. На основе анализа данных, представленных на рис. 3 

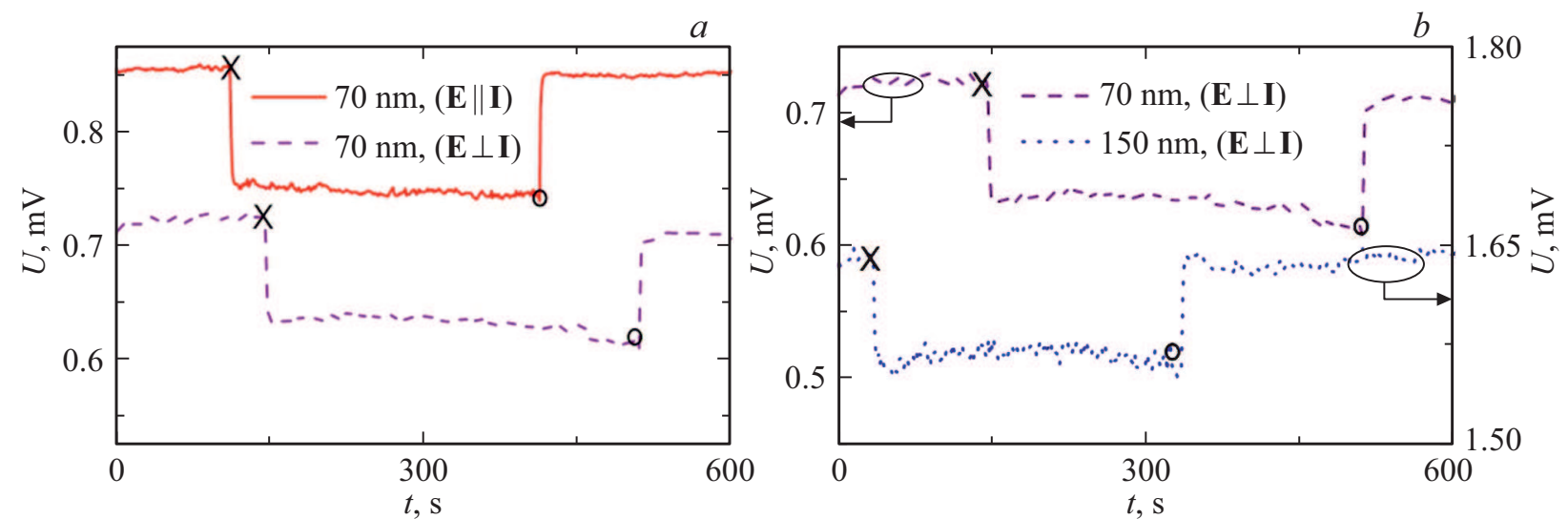

Рис. 3. Зависимости падения напряжения на пленках толщиной 70 и 150 нм от времени: $a-$ для пленки толщиной 70 нм при различных направлениях поляризации; $b-$ для пленок толщиной 70 и 150 нм при перпендикулярном направлении поляризации излучения. На графике обозначены моменты включения $(\times)$ и выключения (о) падающего напряжения.
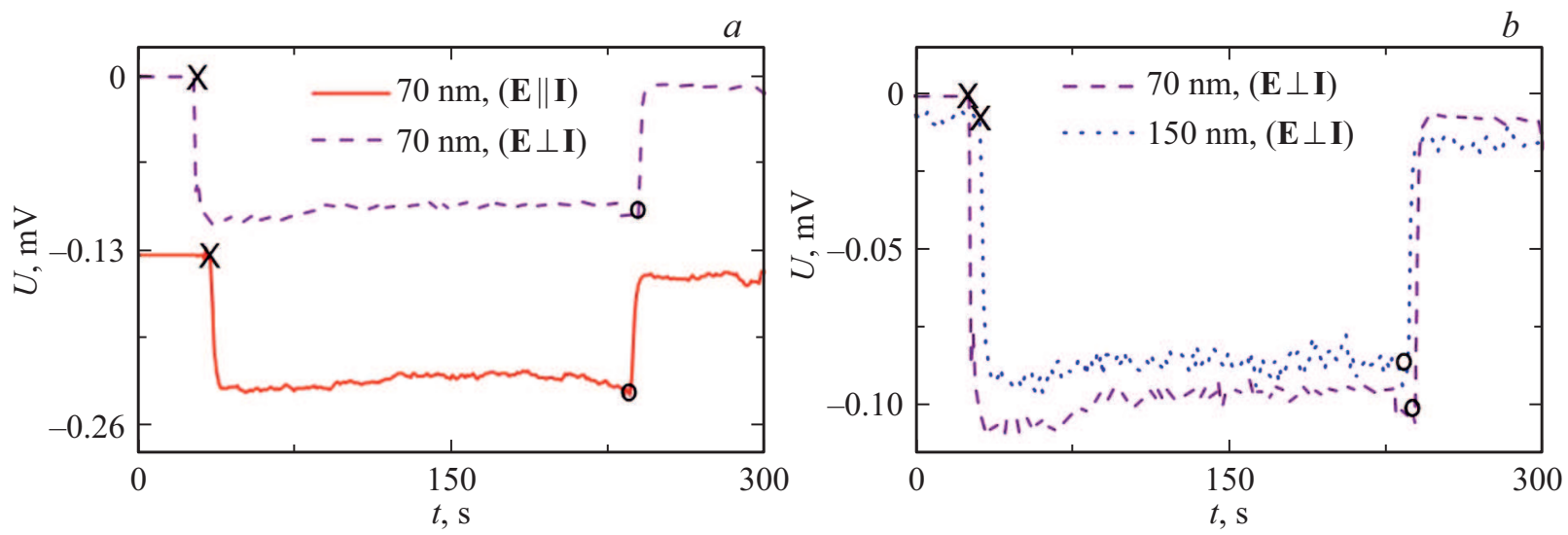

Рис. 4. Зависимость фотоэдс в пленках толщиной 70 и 150 нм от времени. Ток через образец отсутствует: $a-$ для пленки толщиной 70 нм при различных направлениях поляризации; $b-$ для пленок толщиной 70 и 150 нм при перпендикулярном направлении поляризации излучения. На графике обозначены моменты включения $(\times)$ и выключения (о) падающего напряжения.

и 4, можно рассчитать удельную фотопроводимость по разности падения напряжения во всех приведенных случаях, используя формулу

$$
\Delta \sigma=\frac{I}{\Delta U} \cdot \frac{L}{S}
$$

где $\Delta \sigma-$ фотопроводимость, $I-$ сила тока, $\Delta U-$ фотоэдс, $L-$ длина пленки, $S$ - площадь поперечного сечения пленки.

Для пленки 70 нм (рис. 3,a) можно заметить значительное различие в падении напряжения для разного направления поляризации. Если поляризация излучения перпендикулярна направлению тока $(\mathbf{E} \perp \mathbf{I})$, то $\Delta U=-0.089 \mathrm{MB}$, а удельная фотопроводимость составляет $\Delta \sigma_{1}=1.28 \cdot 10^{6}(\mathrm{OM} \cdot \mathrm{M})^{-1}$. Если поляризация излучения параллельна направлению тока $(\mathbf{E} \| \mathbf{I})$, то $\Delta U=-0.101 \mathrm{MB}$, а удельная фотопроводимость $\Delta \sigma_{2}=1.13 \cdot 10^{6}(\mathrm{OM} \cdot \mathrm{M})^{-1}$. Разные результаты $\Delta \sigma_{1}$ и $\Delta \sigma_{2}$ указывают на влияние направления поляризации излучения на подвижность носителей заряда. Такое поведение зависимости от поляризации излучения наблюдалось во всех проведенных экспериментах.

Для пленки толщиной 150 нм (рис. 3,b) и поляризации, перпендикулярной направлению тока $(\mathbf{E} \perp \mathbf{I})$, значение фотоэдс составляет $\Delta U=-0.067 \mathrm{MB}$, a значение удельной фотопроводимости равно $\Delta \sigma_{3}=0.85 \cdot 10^{6}(\mathrm{Oм} \cdot \mathrm{M})^{-1}$. При сравнении величины отклика от пленок разной толщины (см. рис. $3, b$ ) заметно, что величина сигнала от более толстой пленки больше, но значение фотоэдс на $0.022 \mathrm{MB}$ меньше, чем от пленки толщиной 70 нм. Данный эффект может быть связан с небольшим изменением электронного спектра из-за толщины пленки.

На рис. 4, $a$ для пленки толщиной 70 нм приведена зависимость величины фотоэдс от направления поляризации излучения. Если поляризация излучения перпендикулярна оси тока $(\mathbf{E} \perp \mathbf{I})$, то величина фотоэдс составляет $\Delta U=-0.10 \mathrm{MB}$, а поляризация излучения параллельна оси тока $(\mathbf{E} \| \mathbf{I})-\Delta U=-0.22 \mathrm{мB}$. Для пленки толщиной 150 нм (рис. 4,b) при перпендикулярной 


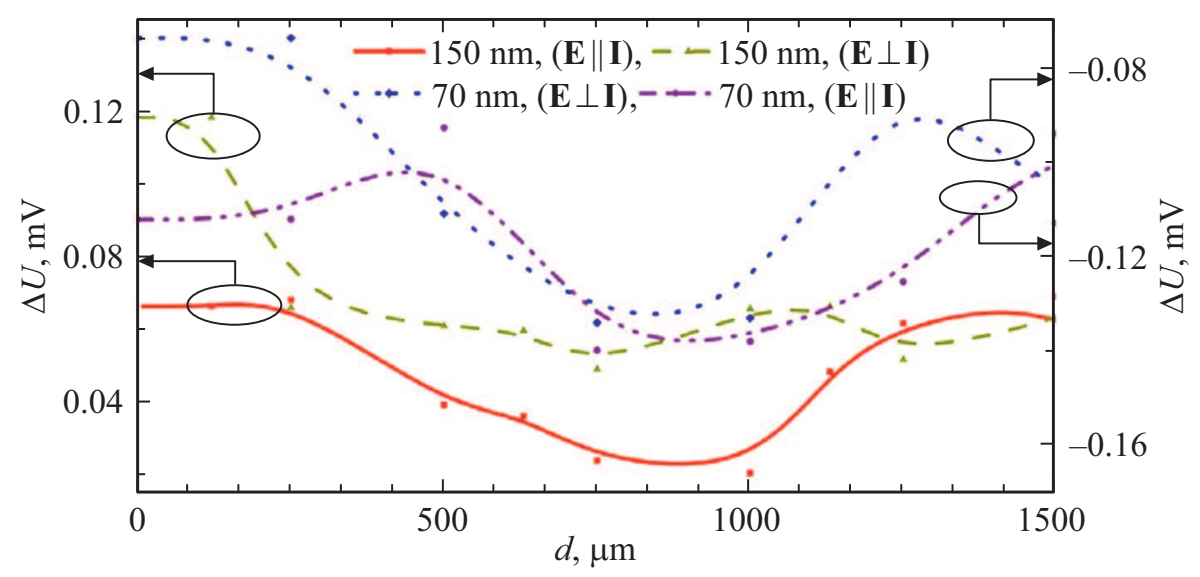

Рис. 5. Зависимость фотоэдс в пленках толщиной 70 и 150 нм от расстояния и поляризации излучения.

поляризации величина фотоэдс составляет $-0.09 \mathrm{MB}$. Рис. 4 хорошо демонстрирует дрейф носителей заряда за напряженностью электрического поля излучения $(\mathbf{E} \| \mathbf{I})$, так как нет другого воздействия на носители заряда, кроме ТГц излучения.

\section{4. Исследование зависимости отклика на ТГц излучение от расстояния между генератором и образцом}

При нормальном падении излучения на пленку $\mathrm{Bi}_{88} \mathrm{Sb}_{12}$ возникает эффект стоячих волн из-за суперпозиции падающей и отраженной волн в пространстве перед детектором. Это приводит к изменению мощности излучения в пространстве между генератором и детектором. Образец фиксировался в заданных точках пространства от источника излучения (см. рис. $2, b$ ), затем в этом положении проводился эксперимент, описанный выше. На рис. 5 хорошо заметно уменьшение фотоэдс в определенной точке пространства, т.е. наблюдается уменьшение энергии, а следовательно, появляется узел стоячей волны. Так как $\sim 25 \%$ излучения отражается от поверхности детектора, необходимо отклонять детектор на небольшой угол, чтобы избежать интерференции волн, которая может привести как к частотной, так и к пространственно-зависимой модуляции детектируемой мощности излучения.

Зависимость индуцированного фототока от ориентации линейно поляризованного излучения для двумерных материалов, к которым с некоторым приближением можно отнести исследуемые в данной работе образцы (ввиду малости отношения толщины пленки к длине волны излучения), носит косинусоидальный характер [17], что также видно на рис. 5. Исходя из предположения о линейной зависимости фототока (фотоэдс) от интенсивности падающего светового излучения можно утверждать, что фототок косинусоидально зависит от угла плоскости поляризации:

$$
I_{\mathrm{ph}}=a \cdot \cos \left(2 \theta+\theta_{0}\right)+I_{0},
$$

где $\theta_{0}$ - начальная фаза.

Таким образом, выбрав $\theta_{0}=0^{\circ}$, можно наблюдать максимальное значение фототока при $\theta_{0}=0^{\circ}$ и минимальное при $\theta=90^{\circ}$.

\section{5. Заключение}

Представленный в работе детектор является многообещающей компактной и недорогой альтернативой существующим детекторам, которые используются для обнаружения ТГц излучения при комнатной температуре. Эта технология может быть масштабно интегрирована в мультипиксельные матрицы детекторов, что позволит создать компактную и экономически выгодную компоновку систем формирования ТГц изображений. Большим преимуществом данных ТГц детекторов является относительно простая масштабируемость частоты обнаружения, благодаря тому что, как терморезистивный эффект, так и термоэлектрический эффект являются широкополосными.

\section{Финансирование работы}

Проведение исследований поддержано грантом РНФ, проект No 19-72-10141.

\section{Конфликт интересов}

Авторы заявляют, что у них нет конфликта интересов.

\section{Список литературы}

[1] P. Bawuah, J.A. Zeitler. Trends Anal. Chem., 139, 116272 (2021).

[2] A. Bandyopadhyay, A. Sengupta. IETE Tech. Rev., 1-19 (2021). 
[3] H. Rahaman, A. Bandyopadhyay, S. Pal, K.P. Ray. IETE Tech. Rev., 1-14 (2020).

[4] S.S. Dhillon, M.S. Vitiello, E.H. Linfield, A.G. Davies, M.C. Hoffmann et al. J. Phys. D: Appl. Phys., 50 (4), 043001 (2017).

[5] F. Sizov. Semicond. Sci. Technol., 33, 123001 (2018).

[6] R.A. Lewis. J. Phys. D: Appl. Phys., 52, 433001 (2019).

[7] A.D. Zaitsev, P.S. Demchenko, D.V. Zykov, E.A. Korotina, E.S. Makarova, I.L. Tkhorzhevskiy, A.S. Tukmakova, N.S. Kablukova, A.V. Asach, A.V. Novotelnova, M.K. Khodzitsky. Appl. Sci., 10 (8), 2724 (2020).

[8] M.K. Khodzitsky, P.S. Demchenko, D.V. Zykov, A.D. Zaitsev, E.S. Makarova, A.S. Tukmakova, I.L. Tkhorzhevskiy, A.V. Asach, A.V. Novotelnova, N.S. Kablukova. Photonics, $8(3), 76$ (2021).

[9] T.-R. Chang, Q. Lu, X. Wang, H. Lin, T. Miller, T.-C. Chiang, G. Bian. Crystals, 9 (10), 510 (2019).

[10] V.M. Grabov et al. J. Thermoelectr., 1, 41 (2009).

[11] В.М. Грабов, Е.В. Демидов, В.А. Комаров, М.М. Климантов. ФТТ, 51 (4), 800 (2009).

[12] В.М. Грабов, Е.В. Демидов, В.А. Комаров. ФТТ, 52 (6), 1219 (2010).

[13] В.М. Грабов, Е.В. Демидов, В.А. Комаров. ФТТ, 50 (7), 1312 (2008).

[14] E. Gerlach, M. Rautenberg. Phys. Status Solidi B, 75 (2), 553 (1976).

[15] В.М. Грабов, Н.П. Степанов. ФТП, 35 (2), 155 (2001).

[16] A. Zaitsev, P.S. Demchenko, M.K. Khodzitsky, E.S. Makarova, A.S. Tukmakova, A.V. Asach, A.V. Novotelnova, N.S. Kablukova. Phys. Status Solidi Rapid Res. Lett., 14(7), 2000093 (2020).

[17] F. Chu, M. Chen, Y. Wang, Y. Xie, B. Liu, Y. Yang, X. An, Y. Zhang. J. Mater. Chem. C, 6 (10), 2509 (2018).

\section{Terahertz radiation detector based on thermoelectric material $\mathrm{Bi}_{88} \mathbf{S b}_{12}$}

\author{
E.S. Makarova ${ }^{1}$, A.V. Asach ${ }^{1}$, I.L. Tkhorzhevskiy ${ }^{1}$, \\ A.D. Sedinin ${ }^{1}$, D.V. Zykov ${ }^{1}$, A.D. Zaitsev ${ }^{1}$, \\ P.S. Demchenko ${ }^{1}$, M.G. Novoselov ${ }^{1}$, V.A. Komarov ${ }^{2}$, \\ A.S. Tukmakova ${ }^{1}$, A.V. Novotelnova ${ }^{1}$, \\ N.S. Kablukova ${ }^{1,3}$, M.K. Khodzitsky ${ }^{1}$ \\ ${ }^{1}$ ITMO University, \\ 197101 St. Petersburg, Russia \\ ${ }^{2}$ The Herzen State Pedagogical University of Russia, \\ 191186 St. Petersburg, Russia \\ ${ }^{3}$ St. Petersburg State University \\ of Industrial Technologies and Design, \\ 191186 St. Petersburg, Russia
}

\begin{abstract}
Terahertz radiation is very promising for visualization, detection and data transfer. Searching for sensitive, fast and compact $\mathrm{THz}$ detector that operates at room temperature is a common subject for these applications. Hence, there is still there is still an issue of searching for new materials for $\mathrm{THz}$ radiation detection. Solid solutions based on thermoelectric bismuth and antimony appear to have significant potential for these applications. In this paper photoresponse of thermoelectric material $\mathrm{Bi}_{88} \mathrm{Sb}_{12}$ has been studied for the first time. Films with thicknesses of 70 and $150 \mathrm{~nm}$ were studied under influence of radiation at frequency of $0.14 \mathrm{THz}$
\end{abstract}

